\title{
Analysis of Traffic Distribution in Cellular Networks
}

\author{
G W Tunnicliffe ${ }^{*}$ A R Murch ${ }^{*}$, A Sathyendran ${ }^{*}$ and P J Smith ${ }^{\#}$ \\ * - BellSouth New Zealand, \# - Victoria University of Wellington, New Zealand \\ BellSouth New Zealand, Level 6, 21 Pitt Street, Private Bag 92161, \\ Auckland, New Zealand Ph. No. +649357 0326, Fax No. +649379 3451, \\ Email : arasaratnam.sathyendran@bellsouth.co.nz
}

\begin{abstract}
Accurate air interface traffic forecasting and dimensioning is of importance in any cellular network for achieving cost and quality requirements. Previous studies of traffic modeling in cellular networks have tended to derive distributions to fit the measured data for the arrival rate and call holding processes or derive expressions for call blocking on the air interface for different handover and channel assignment procedures. In most cases it is assumed that the Erlang B model is not sufficiently accurate and some other call blocking model is required. However, there have not been a large number of studies published on how accurate or otherwise (and under what circumstances) the Erlang B model is in modeling air interface call blocking in practical cellular networks.

In this paper call blocking measurements of the air interface of a "real" cellular network are presented. A statistical analysis is undertaken which shows that the measured data is correctly modeled by Erlang B at a level of significance of 0.05 when the number of channels are greater than 12 and the blocking experienced is greater than $1 \%$. For available channels less than 12 and blocking less than $1 \%$ the Erlang B model overestimates the blocking.
\end{abstract}

\section{Introduction}

Accurate traffic dimensioning is important in any telecommunications network planning and is particularly important for the air interface of cellular networks where this has an effect on the spectral efficiency. Cellular radio networks have traditionally been dimensioned using the Erlang $B$ call blocking model which was originally developed for the fixed network. The application of the Erlang B call blocking model assumes: that there are a large number of users, that the arrival process follows a Poisson process, that there is full availability (i.e. an arriving call can use any free circuit) and that lost calls are cleared (i.e. they leave the system and do not re-attempt). However, in a cellular network mobiles within the coverage area are in general only able to seize circuits on the cell site serving that area. In cellular networks blocked calls may be reattempted in neighboring cells (provided signal strength requirements are met). Also full availability may not be possible if a priority access scheme has been implemented.
Claims have been made about the appropriateness or otherwise of Erlang B to model call blocking on the air interface. In [1] it is stated that "the difference between Erlang B model and measured cellular air interface blocking can be up to 15 percent, however the Erlang B model is still considered as a good model for obtaining useful estimates". However, few studies have been published detailing how appropriate Erlang B is in modeling call blocking on the air interface. This paper presents an analysis of the appropriateness or otherwise of Erlang $\mathrm{B}$ for modeling call blocking on the air interface.

A number of studies have been done previously to study the air interface traffic of cellular networks [2-8]. In [2] probability distributions have been derived for channel holding time. In [3] traffic performance was considered in FDMA, TDMA and CDMA networks. In [4] blocking expressions were derived for different handover procedures. In [5] blocking expressions were derived for single cell cellular networks and were extended to multi-cell cellular networks in [6]. Generally the validity of Erlang B distribution to model cellular network traffic has been questioned in these studies. Improved and usually complicated models have been presented to estimate the blocking performance.

From a network operators point of view it is difficult to derive blocking models based on the new distributions suggested in many of these studies. Similarly it is also difficult to use the complicated blocking expressions as it would require the knowledge of the nature of traffic in their particular network. I1 is advantageous to use existing tables or modified versions of established tables without requiring to do an in-depth analysis This is the motivation behind this study.

This paper initially compares the call arrival and holding time distributions from Erlang B model with that measured from the network (Section II). Then a detailed statistical analysis comparing measurement data of air interface blocking versus offered traffic with those calculated using Erlang B is presentec (Section III). Conclusions from the study are presented ir Section IV.

\section{Call Arrival and Holding Time Distributions}

This section presents call arrival rate and holding time measurement data on the air interface and where appropriate 
compares it with assumptions that underly Erlang B. The traffic measurements presented here have been taken from a cellular network which has approximately 450 cells and 10,000 air interface channels. Approximately $10 \%$ of the cells serve an area of $5 \mathrm{~km}^{2}$ or less, another $20 \%$ of the cells serve an area of $80 \mathrm{~km}^{2}$ or less, while the remaining $70 \%$ serve areas of varying size of up to $2,000 \mathrm{~km}^{2}$. Approximately $80 \%$ of the traffic is generated on the $30 \%$ of the cells which serve areas of $80 \mathrm{~km}^{2}$ or less. Also, blocking calls are reattempted in a neighbouring cell if it is possible, and no priority access or other means of limiting full availability is implemented.

\section{A. Arrival Rate Distribution.}

Erlang $\mathrm{B}$ assumes the call arrival process is Poisson. The Poisson distribution is given by [9]:

$$
\mathrm{P}(n)=\frac{(\lambda t)^{n}}{n !} \exp (-\lambda t),
$$

where $\lambda$ is the mean arrival rate and $P(n)$ is the probability of $n$ arrivals in time $t$.

Figures 1 and 2 present measurements for call arrivals at the air interface (inbound and outbound calls) of particular cells and plots the Poisson distribution for $\lambda$ derived from the measurement data. The measurements were made by counting the number of arrivals in a 10 second interval over a typical busy hour. The measurement data presented in figures 1 and 2 are from two different cells which serve areas of $5 \mathrm{~km}^{2}$ or less. Both sites carried approximately 15 Erlangs of traffic (during the busy hour). The data presented in Figures 1 and 2 are both scaled by the total number of arrivals.

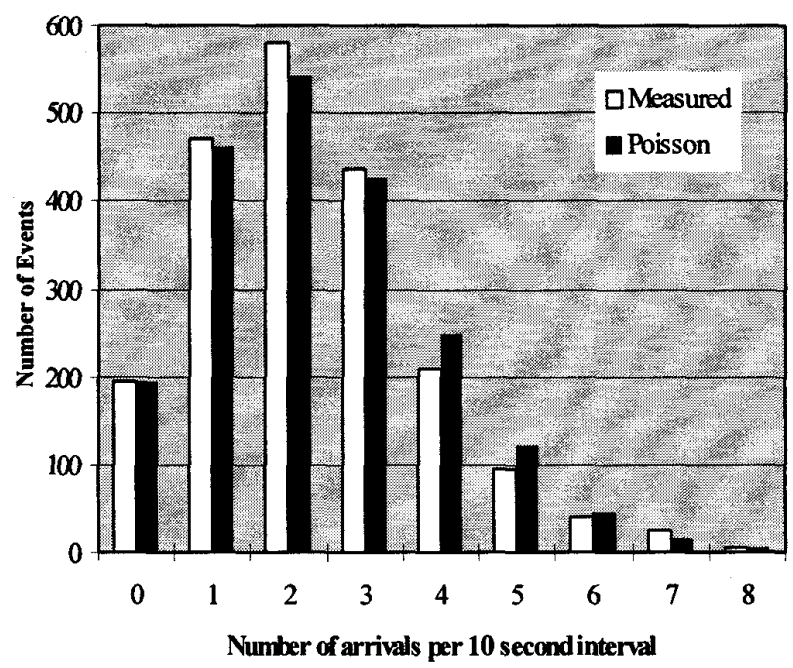

Figure 1: Measurement data compared to the Poisson distribution for a cell serving an areas of less than $5 \mathrm{~km}^{2}$.

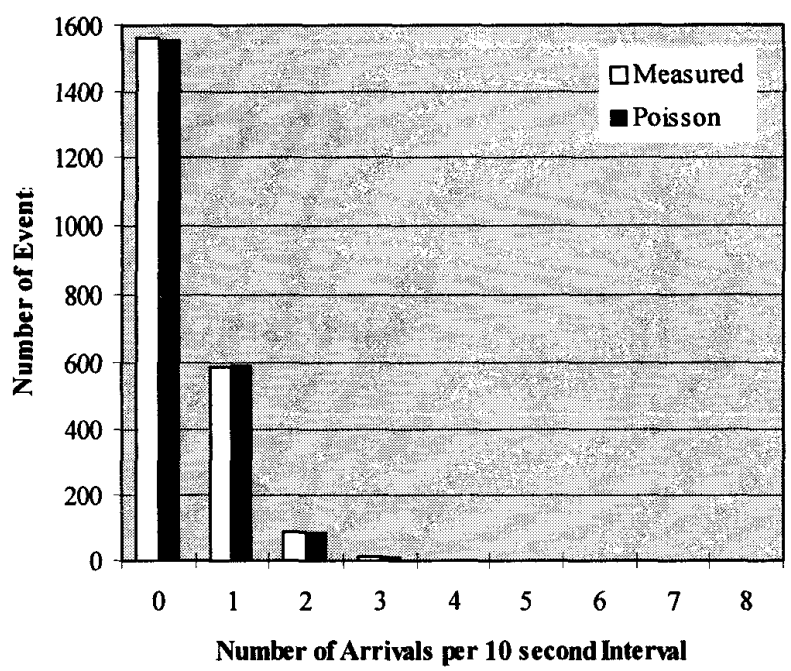

Figure 2: Measurement data compared to the Poisson distribution for a cell serving an areas of less than $5 \mathrm{~km}^{2}$.

Invoking the Chi-squared test for goodness of fit to Figures 1 and 2 , the hypothesis that the measured arrival process is different from Poisson, is rejected at a level of significance of $\alpha=0.05$. Although we have not studied all sites in the network the sites selected for presentation here were not specially selected.

\section{B. The Holding Time Distribution.}

The call holding time distribution in fixed networks tends to be an exponential distribution. The exponential distribution is given by [10]:

$$
\mathrm{P}(x)=A \exp [-A x]
$$

where $\mathrm{A}$ is the mean holding time and $\mathrm{P}(x)$ is the probability that the holding time is of value $x$. Taking the natural $\log$

$$
\ln (\mathrm{P}(x))=\ln (A)-A x .
$$

Equation (3) is a straight line. Figure 3 presents the call holding time measurements. The holding time measurements were obtained by sampling the channel occupancy at 10 second intervals.

In Figure 3 two straight lines which best fit the measurement data are plotted. The break point in this graph indicates that the holding time distribution is actually the result of at least two processes (unlike the fixed network). This has been observed by others and is generally considered to be the result of calls which originate and terminate within the same cell (e.g. stationary user) and calls which have been handed over to and from the cell (e.g. a user in a car). It is worth noting that the Erlang $B$ is not sensitive to the call holding time distribution. 


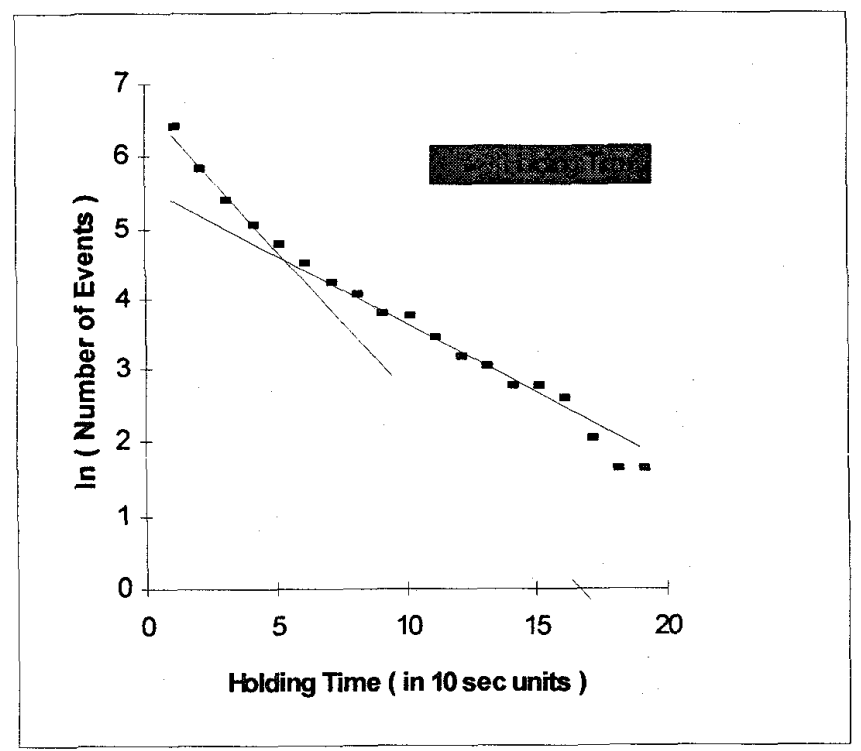

Figure 3: Log of the call holding time distribution.

\section{Network Blocking Performance.}

The purpose of this section is to statistically compare the measured air interface call blocking with Erlang B.

Figures 4, 5, and 6 present measured data of air interface call blocking versus offered traffic for a 6 month period from all sites in the network. Each point represents a measurement from the busy hour. The offered traffic and blocking probability were estimated from the busy hour carried traffic and busy hour congestion time. Figures 4,5 and 6 are plots for cell sites with 7,14 and 22 available channels respectively. The blocking probability versus offered traffic given by Erlang $B$ is also plotted.

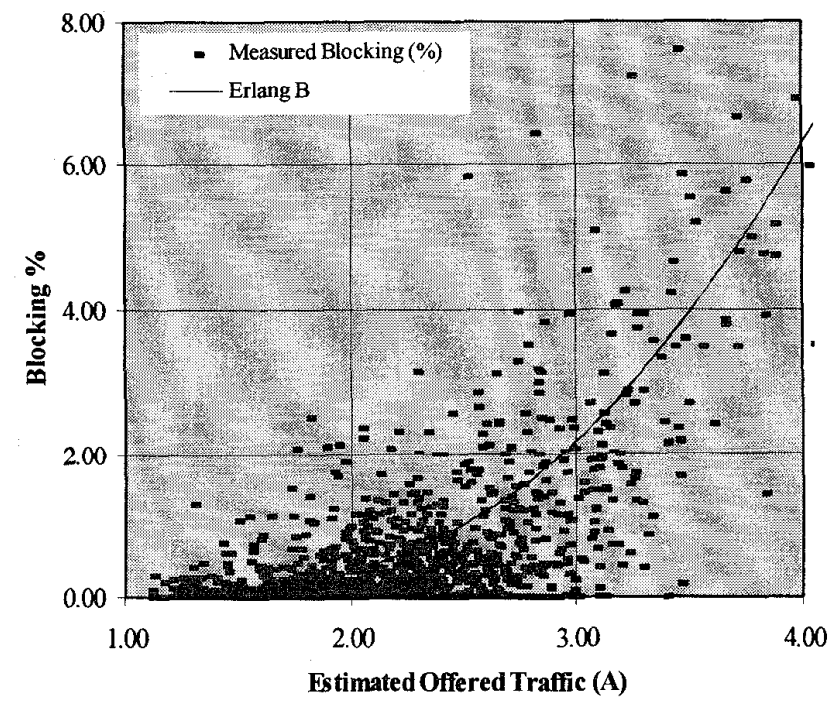

Figure 4: Measured and Erlang B blocking versus offered traffic for a 7 channel site.

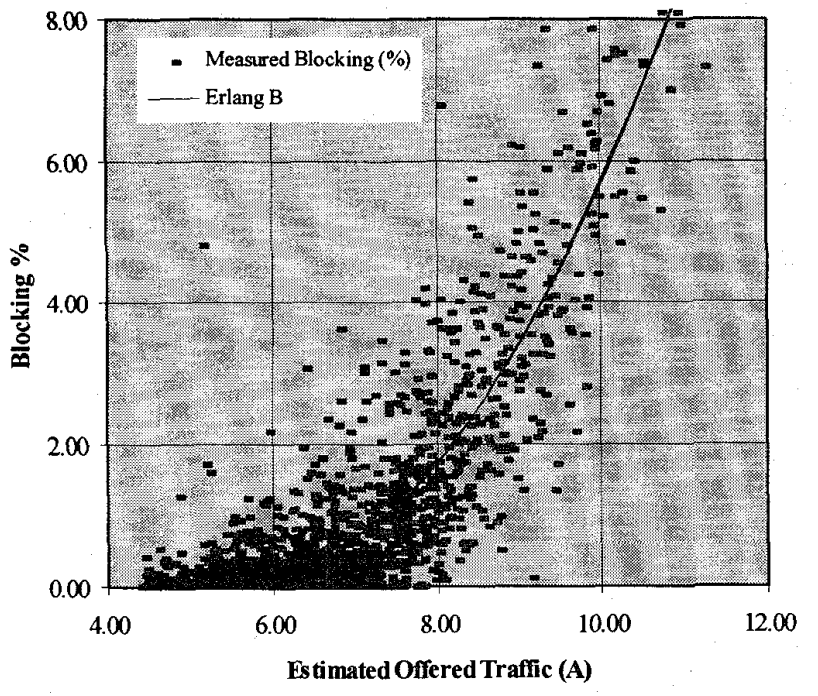

Figure 5: Measured and Erlang B blocking versus offered traffic for a 14 channel site.

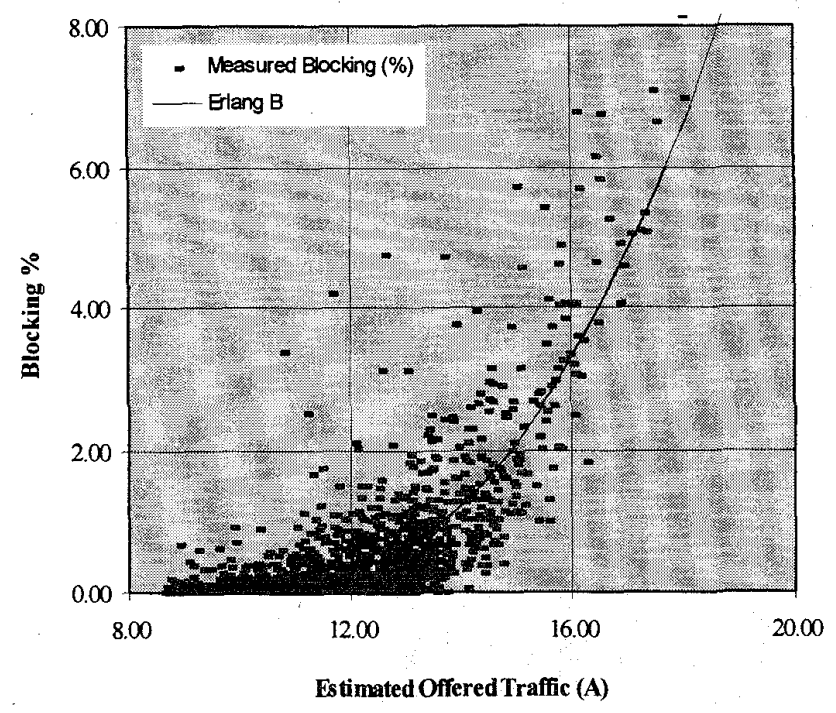

Figure 6: Measured and Erlang B blocking verses offered traffic for 22 channel site.

By inspection Figures 4, 5 and 6 shows that in some sense at least Erlang $B$ approximates the measured results. However, it is not clear how well Erlang B approximates the measured results. The root mean squared error and the mean error between Erlang $\mathrm{B}$ and the measured data is tabulated in Table 1 . 
Table 1: The mean squared error and the mean error between Erlang B and the measured data.

\begin{tabular}{|c|c|c|}
\hline No. of Channels & Root Mean Squared Error & Mean Error \\
\hline 7 & 0.49 & 0.15 \\
\hline 14 & 0.63 & 0.03 \\
\hline 22 & 0.64 & 0.00 \\
\hline
\end{tabular}

The root mean squared values in Table 1 indicate that there is some discrepancy between Erlang B and the measured data. To compare the measurement data with Erlang B, the measured data is divided into 0.2 Erlang bins spaced at 0.5 Erlangs. The number of measurement points that falls within the 0.2 Erlang bin (ie. sample size), the confidence interval of the mean call blocking in the bin (the confidence interval uses a level of significance of $\alpha=0.05$ ) and blocking calculated using Erlang $B$ (in the Erlang $B$ calculation the offered traffic value used in the calculation is the value corresponding to the center of the bin) are tabulated in Tables 2,3 and 4 corresponding to the measurement data presented in Figures 4,5 and 6. The same information contained in Tables 2,3 and 4 is visually presented as plots in Figure 7.

Table 2 : $\quad$ The measurement data divided into 0.2 Erlang bins spaced at 0.5 Erlangs for the 7 channel site measurement data presented in Figure 4.

\begin{tabular}{|c|c|c|c|}
\hline Bin & Sample Size & Confidence Interval & Erlang B \\
\hline $1.4-1.6$ & 714 & $(0.012,0.026)$ & 0.074 \\
\hline $1.9-2.1$ & 375 & $(0.123,0.195)$ & 0.339 \\
\hline $2.4-2.6$ & 141 & $(0.412,0.672)$ & 0.989 \\
\hline $2.9-3.1$ & 54 & $(0.927,1.531)$ & 2.172 \\
\hline $3.4-3.6$ & 19 & $(2.986,4.596)$ & 3.986 \\
\hline
\end{tabular}

Table 3: The measurement data divided into 0.2 Erlang bins spaced at 0.5 Erlangs for the 14 channel site measurement data presented in Figure 5.

\begin{tabular}{|c|c|c|c|}
\hline Bin & Sample Size & Confidence Interval & Erlang B \\
\hline $4.4-4.6$ & 444 & $(0.002,0.01)$ & 0.018 \\
\hline $4.9-5.1$ & 330 & $(0.006,0.014)$ & 0.047 \\
\hline $5.4-5.6$ & 270 & $(0.047,0.089)$ & 0.108 \\
\hline $5.9-6.1$ & 195 & $(0.088,0.162)$ & 0.224 \\
\hline $6.4-6.6$ & 183 & $(0.23,0.336)$ & 0.415 \\
\hline $6.9-7.1$ & 98 & $(0.391,0.645)$ & 0.711 \\
\hline $7.4-7.6$ & 96 & $(0.897,1.295)$ & 1.140 \\
\hline $7.9-8.1$ & 54 & $(1.314,1.95)$ & 1.709 \\
\hline $8.4-8.6$ & 32 & $(2.409,3.333)$ & 2.453 \\
\hline $8.9-9.1$ & 26 & $(3.277,4.329)$ & 3.347 \\
\hline $9.4-9.6$ & 16 & $(3.404,4.964)$ & 4.441 \\
\hline
\end{tabular}

Table 4 : $\quad$ The measurement data divided into 0.2 Erlang bins spaced at 0.5 Erlangs for the 22 channel site measurement data presented in Figure 6.

\begin{tabular}{|c|c|c|c|}
\hline Bin & Sample Size & Confidence Interval & Erlang B \\
\hline $8.9-9.1$ & 268 & $(0.002,0.01)$ & 0.011 \\
\hline $9.4-9.6$ & 226 & $(0.003,0.017)$ & 0.021 \\
\hline $9.9-10.1$ & 186 & $(0.024,0.024)$ & 0.040 \\
\hline $10.4-10.6$ & 144 & $(0.02,0.048)$ & 0.072 \\
\hline $10.9-11.1$ & 153 & $(0.058,0.11)$ & 0.119 \\
\hline $11.4-11.6$ & 122 & $(0.081,0.169)$ & 0.194 \\
\hline $11.9-12.1$ & 125 & $(0.175,0.291)$ & 0.303 \\
\hline $12.4-12.6$ & 87 & $(0.316,0.518)$ & 0.454 \\
\hline $12.9-13.1$ & 81 & $(0.513,0.755)$ & 0.658 \\
\hline $13.4-13.6$ & 67 & $(0.629,0.983)$ & 0.933 \\
\hline $13.9-14.1$ & 29 & $(0.777,2.239)$ & 1.241 \\
\hline $14.4-14.6$ & 26 & $(1.238,1.87)$ & 1.622 \\
\hline $14.9-15.1$ & 15 & $(1.647,3.009)$ & 2.084 \\
\hline
\end{tabular}




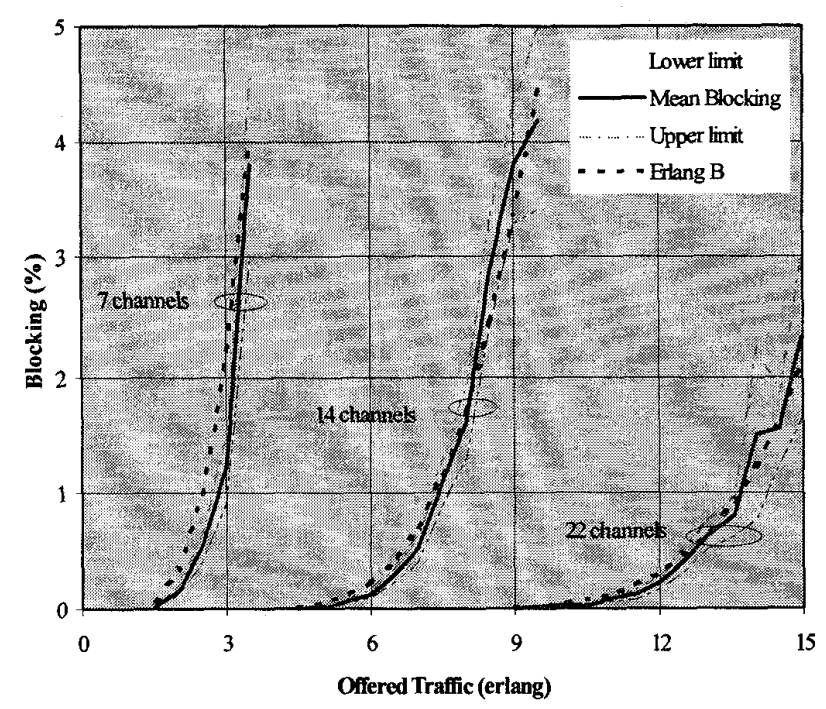

Figure 7: Offered traffic versus blocking plotted for the measured data and Erlang B. The measurement data is divided into 0.2 Erlang bins spaced 0.5 Erlangs apart. The upper and lower limit of the confidence interval calculated for each bin is plotted.

Figure 7 is useful in visually indicating where the Erlang B plot falls inside or outside the confidence interval of each of the measurement bins. Where the Erlang B plot falls inside the measured data confidence interval indicates that Erlang B from a statistical view cannot be rejected as the process underlying the measured data. Figure 7 shows that Erlang B falls within the measured data confidence interval more often when there are more channels (greater than 12) per site and when blocking is above $1 \%$. Conversely figure 7 shows that Erlang $B$ falls outside the measured data confidence interval when there is fewer channels per site and when blocking is less than $1 \%$.

\section{Conclusions}

From the measurements of the cellular network presented in this paper Erlang B is an appropriate model for calculating call blocking on the air interface when the number of channels per cell site is greater than about 12 and where the call blocking is greater than about $1 \%$.

Where Erlang B is not appropriate (i.e. channels less than about 12 and blocking less than 1\%) Erlang B over estimates call blocking (ie. blocking worse than what it really is). This indicates that cellular traffic in cells with a small number of channels is smoother than assumed by Erlang B.

In this paper primarily the mean of the measured data was compared with that of Erlang B model. Further analysis is needed to study how well the variance compares with the Erlang B model.

\section{Acknowledgement}

The authors would like to thank Gary Woolf for providing the measurement data.

\section{References}

[1] William C.Y. Lee " Mobile Cellular Telecommunications Systems " McGraw Hill International Editions, 1989.

[2] Chris Jedrzycki and Victor C.M.Leung "Probability Distribution of Channel Holding Time in Cellular Telephoney Systems " Proceedings of IEEE 44th VTC, pp. 247-251, Sweden 1994.

[3] David E. Everitt, "Traffic Engineering of the Radio Interface for Cellular Mobile Networks " Proceedings of IEEE. VOL 82, NO 9. pp. 1371-1382, September 1994.

[4] Stephen S. Rappaport, and Lon-rong $\mathrm{Hu}$, "Microcellular Communication Systems with Hierarchical Macrocell Overlays: Traffic Performance Models and Analysis" Proceedings of IEEE, pp. 1383-346, VOL 82, NO 9. September 1994.

[5] S H Bakry and MH Ackroyd, "Teletraffic analysis for single-cell mobile radio telephone systems," IEEE Trans. Commun., vol. COM-29, pp. 298-304, March 1981.

[6] S H Bakry and MH Ackroyd, "Teletraffic analysis of multicell mobile radio telephone systems," IEEE Trans. Commun., vol. COM-30, pp. 1905-1909, August 1982.

[7] Bijan Jabbari " Teletraffic Aspects of Evolving and NextGeneration Wireless Communication Networks" IEEE Pers. Commun., Dec 1996.

[8] Roch A. Guerin, "Channel Occupancy Time Distribution in a Cellular Radio System" IEEE Trans. Vech. Technol, vol. VT-35. NO. 3, pp. 89-99, August 1987.

[9] D Bertsekas and $R$ Gallager, "Data Networks," Englewood Cliffs, NJ: Prentice-Hall, second edition, 1987.

[10] A Papoulis, "Probability, Random Variables, and Stochastic Processes," third edition, McGraw-Hill, 1991. 